\title{
Abordaje actual de las metástasis hepáticas sincrónicas de origen colorrectal: ¿secuencial, simultáneo o reverso?
}

\author{
Cristian A. Angeramo ${ }^{1}$, Darío Ramallo, ${ }^{1,2}$, Lucas Mc Cormack ${ }^{1}$ \\ ${ }^{1}$ Sección Cirugía hepática y Trasplante. Servicio de Cirugía General. Hospital Alemán. CABA, Argentina. \\ ${ }^{2}$ Fellow de Cirugía hepato-bilio-pancreática y Trasplante de órganos abdominales. Hospital Alemán. CABA, Argentina.
}

De acuerdo con el registro del Instituto Nacional del Cáncer, en Argentina el cáncer colorrectal (CCR) es el tercero en incidencia y el segundo de mayor mortalidad a nivel nacional. ${ }^{1}$ Más del $50 \%$ de los pacientes con CCR desarrollará enfermedad metastásica durante el curso de su enfermedad. ${ }^{2}$ Por definición, todas las metástasis, en cualquier órgano que asienten (detectables o no), son sincrónicas porque surgen como consecuencia de la diseminación por vía linfática, vascular o traumática (instrumentación o ruptura espontánea) del tumor primario. Por consenso y para una mejor categorización de las mismas, se han definido como metástasis hepáticas sincrónicas (MHS) a aquellas diagnosticadas antes o al momento del diagnóstico del tumor primario. Las metástasis hepáticas metacrónicas (MHM) son aquellas que no se detectan en la estadificación inicial del CCR y son clasificadas en tempranas o tardías según si las lesiones son detectadas antes o después de los 12 meses posteriores al diagnóstico del tumor primario, respectivamente. ${ }^{2}$

Cada uno de los pacientes con metástasis hepáticas (MH) de CCR deberá ser discutido en forma interdisciplinaria teniendo en cuenta un aspecto sistémico de la enfermedad, considerando variables del paciente (performance status, comorbilidades, entorno psicosocial), del tumor primario (resecabilidad local y sintomatología) y de su compromiso tumoral a nivel hepático. En este último aspecto, los pacientes deben cumplir criterios de resecabilidad hepática no solo técnica (resección tumoral con márgenes negativos e hígado remanente futuro con adecuado volumen y funcionalidad), sino también oncológica (enfermedad extrahepática ausente o resecable, tumor primario controlado o resecado, ausencia de progresión tumoral bajo quimioterapia sistémica). ${ }^{3}$ Desde el punto de vista quirúrgico, independientemente del abordaje laparoscópico o convencional, en la resección hepática de pacientes con MH se deben aplicar los conceptos modernos de cirugía preservadora de parénquima para dismi-

Los autores declaran ausencia de conflictos de interés.

Lucas Mc Cormack

Imccormack@hospitalaleman.com

Recibido: julio de 2020. Aceptado: agosto de 2020. nuir la extensión innecesaria de la cirugía hepática. De esta manera se procede a una cirugía ahorrativa de hepatocitos funcionantes, es decir, una cirugía que preserve el máximo de parénquima hepático no tumoral. Recientes publicaciones demuestran que esta estrategia presenta menores índices de complicaciones mayores e insuficiencia hepática postoperatoria, similares resultados oncológicos comparada con resecciones mayores y finalmente incrementa la posibilidad de una nueva resección futura en caso de recurrencia en el hígado (50-60 \% tendrán una recurrencia hepática). ${ }^{4}$

La estrategia quirúrgica de las MHM resecables es siempre "secuencial" (primer tiempo: cirugía CCR; segundo tiempo: hepatectomía). En esta población, la cirugía colorrectal ya ha sido realizada al momento del diagnóstico y la cirugía hepática se presenta para su ejecución en una segunda etapa de la evolución de la enfermedad. Lamentablemente, la resecabilidad de las MH de CCR utilizando un abordaje hepático moderno y agresivo está limitada al 30-50 \% de los casos. En aquellos casos con MH no resecables, la terapia sistémica es el tratamiento adecuado con la intención de prolongar la sobrevida y mejorar la calidad de vida de los pacientes. En aquellos casos con MHS resecables, a diferencia de lo que sucede con la enfermedad metacrónica, la decisión suele ser de mayor complejidad porque se presentan 3 potenciales opciones de tratamiento quirúrgico con intención curativa: el abordaje secuencial, el simultáneo o el reverso.

Hay dos variables importantes que condicionan la estrategia a utilizar en aquellos pacientes con MHS que son potencialmente resecables: la sintomatología relacionada al tumor primario y la extensión de la resección hepática. Por otro lado, hay dos reglas que deberían ser cumplidas en todo paciente con MHS. Primera regla: todo CCR sintomático debe ser inicialmente tratado de urgencia. Segunda regla: se debe priorizar, en centros con disponibilidad de equipos entrenados en cirugía hepática y colorrectal, el abordaje "simultáneo" (resección del CCR y de las MHS en un único tiempo quirúrgico) para evitar el efecto sumatorio de la morbilidad de dos cirugías mayores con anestesia general. Es importante re- 
saltar que la cirugía simultánea no estaría recomendada en algunas situaciones; pacientes con múltiples comorbilidades con alto riesgo quirúrgico, necesidad de una resección colónica compleja, presentación con obstrucción o perforación colónica, necesidad de una resección hepática mayor ( $\geq 3$ segmentos), o en pacientes con tumores de recto medio o bajo que requieren tratamiento neoadyuvante con radioterapia.

Entre un 20-40\% de los pacientes portadores de MHS irresecables al diagnóstico, son rescatados y transformados en resecables por la administración de nuevos esquemas de quimioterapia de conversión basados en FOLFIRI o FOLFOXIRI con o sin la asociación de un anticuerpo monoclonal. En este subgrupo de pacientes convertidos o en aquellos que debutan con alta carga tumoral hepática y necesidad de hepatectomía compleja con una resecabilidad técnica muy "al límite", la recomendación sería la de innovar con el abordaje "reverso" (primer tiempo: cirugía hepática; segundo tiempo: cirugía CCR). Esta estrategia permitiría evitar la eventual progresión hepática que podría resultar de un abordaje secuencial con requerimiento de un intervalo prolongado entre ambas cirugías. El concepto del abordaje reverso sería el de resolver primero el foco de mayor compromiso tumoral y luego la cirugía del CCR asintomático. Una vez resuelta exitosamente la enfermedad hepática, se puede optar por realizar o no quimioterapia sistémica durante el intervalo hasta la cirugía definitiva del primario. Finalmente, en pacientes portadores de cáncer de recto medio o bajo con MHS que requieran cirugía hepática mayor, se podría iniciar el tratamiento sistémico y radioterapia del recto y luego realizar la hepatectomía en el lapso de tiempo que habitualmente se requiere entre la radioterapia y la cirugía rectal. ${ }^{2}$
El abordaje "secuencial" clásico (primer tiempo: cirugía CCR; segundo tiempo: cirugía hepática) sigue teniendo vigencia particularmente para pacientes con CCR y MHS que no cumplan los criterios de abordaje simultáneo. Esta estrategia debe ser aplicada a pacientes con MHS y presencia de CCR sintomático (hemorragia clínicamente relevante, perforación u obstrucción colónica). En las perforaciones colónicas, la resección del tumor primario debe ser realizada siempre que sea posible. En el caso de la obstrucción colónica la cirugía resectiva será de elección, aunque actualmente es materia de debate la utilización de stents colónicos en lesiones localizadas principalmente en colon sigmoides o colon izquierdo. ${ }^{5}$ Luego de realizada la cirugía resectiva del CCR, la cirugía hepática sería efectuada después de un intervalo variable de 1-3 meses con o sin la asociación de una reconstrucción del tránsito colónico y con o sin la administración de quimioterapia sistémica previa, según la situación clínica del paciente y la decisión interdisciplinaria. El abordaje secuencial tiene como ventaja adicional una mejor selección de los pacientes con MHS mediante la evaluación empírica de la "biología" tumoral, evitando la cirugía hepática en pacientes con enfermedad en progresión en el corto plazo de intervalo de observación.

En conclusión, la selección de la estrategia secuencial, simultánea o reversa en pacientes con MHS de CCR es una decisión compleja que requiere de la inteligencia colectiva de un equipo interdisciplinario que incluya cirujanos hepáticos y colorrectales, oncólogos clínicos, endoscopistas, patólogos e imagenólogos. Cada una de las decisiones debe ser discutida y consensuada para luego ser adaptadas a cada paciente en particular, con el objetivo de obtener los mejores resultados clínicos-quirúrgicos y oncológicos a corto y largo plazo.

\section{REFERENCIAS}

1. Guía para equipos de atención primaria de la salud. Programa Nacional de Prevención y Detección Temprana del Cáncer Colorrectal (PNCCR) Instituto Nacional del Cáncer (INC); 2015. http://www.msal.gob.ar/images/stories/bes/ graficos/0000000899cnt-2016-10-28-guia_ccr_aps.pdf

2. Adam R, de Gramont A, Figueras J, Kokudo N, Kunstlinger F, Loyer E, et al. Managing synchronous liver metastases from colorectal cancer: a multidisciplinary international consensus. Cancer Treat Rev 2015;41:729-41.

3. Adams RB, Aloia TA, Loyer E, Pawlik TM, Taouli B, Vauthey JN.
Selection for hepatic resection of colorectal liver metastases: expert consensus statement. HPB (Oxford) 2013;15:91-103.

4. Torzilli G, McCormack L, Pawlik T. Parenchyma-sparing liver. Int J Surg 2020; S1743-9191(20)30346-0. Available from: http://dx.doi. org/10.1016/j.ijsu.2020.04.047.

5. Pisano M, Zorcolo L, Merli C, Cimbanassi S, Poiasina E, Ceresoli M, et al. 2017 WSES guidelines on colon and rectal cancer emergencies: obstruction and perforation. World J Emerg Surg 2018;13:36. 